\title{
On the Formation of Lithium Emission Lines in Nova Shells
}

\author{
Marcos Diaz \\ Instituto Astronômico e Geofísico - Universidade de São Paulo, \\ 04301-904, São Paulo, SP, Brazil
}

\begin{abstract}
The role of nova outbursts in the galactic lithium production is still controversial. Photoionization model calculations of nova shells are presented in this contribution, aiming to identify the optimal postoutburst phase for detecting lithium emission lines from nova ejecta which may confirm its lithium enhancement. Predictions for the Li I $\lambda 6708$ $\AA$ flux are made as a function of the envelope geometry, its physical parameters and properties of the central ionizing source.
\end{abstract}

\section{Introduction}

Over the last two decades theoretical models of thermonuclear runaways (TNR) in novae have predicted a physical scenario where significant amounts of ${ }^{7} \mathrm{Li}$ can be formed (Starrfield et al. 1978). According to recent hydrodynamic calculations, the convective time-scale in the core-envelope interface was found to be shorter than the life-time of ${ }^{7} \mathrm{Be}$, allowing ${ }^{7} \mathrm{Li}$ enhancements of $10^{2}$ to $10^{3}$ with respect to solar values (Hernanz et al. 1996). Although lithium absorption lines have been detected in the secondary spectrum of a few black hole binaries, there is no observational confirmation of the nova ${ }^{7} \mathrm{Li}$ production mechanism in cataclysmic binaries. Depending on the conditions during outburst such a process may result in a significant contribution to the Galaxy ISM lithium abundance.

\section{Nova Shell Models}

The line forming region in the nova expanding shell evolves rapidly and therefore requires a time-resolved description. On the other hand, the intrinsic scatter in nova basic parameters has to be taken into account when searching for possible episodes of lithium line production. Our nova envelope models share many common fixed parameters which are described bellow. Average chemical abundances for non-ONeMg novae (Gehrz et al. 1998) were employed with a constant ${ }^{7} \mathrm{Li}$ enhancement of 1000 times the solar value. Typical maximum and minimum nova shell expansion velocities of 600 and $1000 \mathrm{~km} / \mathrm{s}$ define the shell geometry as a function of time in the impulsive ejection approximation. The mass distribution inside the envelope is defined by a radial power density law with index $\alpha=-0.9$ (Diaz et al. 1995). An extensive model grid containing more than 13000 points was used to study the complex line flux behavior over a wide range of shell masses $\left(10^{-6} \leq M_{\text {shell }} \leq 10^{-4} M_{\odot}\right)$, time after maximum $(\leq 5000$ 
days), and central source temperatures $\left(80000 \leq T_{\text {eff }} \leq 350000 K\right)$ and luminosities $\left(10^{35} \leq L_{c} \leq 10^{38.5} \mathrm{erg} / \mathrm{s}\right)$. The ionizing spectrum is approximated by NLTE, high-gravity stellar model atmospheres (Rauch 1997). Finally, the effect of gas condensations in the nova envelope (Williams 1992) was investigated by randomly adding Gaussian globules to the background density law. For a few control points the line transfer was calculated under the Sobolev approximation yielding unimportant discrepancies in the line ratios when compared to the static case. All the calculations described in this contribution were made by the code RA3D which uses CLOUDY (Ferland et al. 1998) as a subroutine.

\section{Observational Perspectives}

The strongest emission line in the optical ( $\mathrm{Li} I \lambda 6707 \AA$ ) is mainly formed by collisional excitation from the ground state in the shell environment. The simulations indicate a well defined set of constraints for guiding deep spectroscopic observations of nova remnants. Our results suggest that the 6707 line emission is strongly suppressed in luminous and super-eddington remnants (i.e. in very fast novae) and increases for lower luminosity remnants $\left(L_{c} \lesssim 10^{36.7} \mathrm{erg} / \mathrm{s}\right)$. In the case of hot sources, which usually display high ionization spectra, such a emission is restricted to $\sim 18$ months after the visual maximum. Relatively low temperature sources $\left(T_{\text {eff }} \lesssim 130,000 \mathrm{~K}\right)$ may produce significant 6707 line flux during an extended period of time after maximum ( $\$ 3.0 \mathrm{yr}$.). Such a timescale opens the possibility for spatially resolved diagnostic of nearby envelopes (Diaz 1998). Novae with low ejection efficiencies (i.e. having low nebular densities) present smaller emissivities while shells with mass above $10^{-5} M_{\odot}$ produce stronger emission episodes along their evolution. Due to the low first ionization potential of lithium, most of the line originates in almost neutral gas. This explains the significant effect seen when the fractional mass in globules is increased to $z 30 \%$. These clumpy models suggest that the $6707 / \mathrm{H} \beta$ ratio may raise by one order of magnitude in a heterogeneous medium, depending on condensation properties. In general, the predicted 6707 relative flux is a small quantity reaching, in favorable situations, values around $10^{-3} \times \mathrm{H} \beta$. The maximum expected fluxes are close to $10^{-15} \mathrm{erg} . \mathrm{s}^{-1} . \mathrm{cm}^{-2}$ for an unabsorbed remnant at $1.0 \mathrm{kpc}$.

\section{References}

Diaz, M. 1998, in Science with Gemini, eds. Barbuy, Lapaset, Baptista, \& Fernandes, (Santa Catarina:UFSC).

Diaz, M., Williams, R., Phillips, M., \& Hamuy, M. 1995, MNRAS, 277, 959.

Ferland, G. 1998, Korista, K., Vernet, D., Fergunson, J., Kingdon, J., \& Verner, E. 1998, PASP, 110, 761.

Gehrz, R., Truran, J., Williams, R., \& Starrfield, S. 1998, PASP, 110, 3.

Hernanz, M., Jose, J., Coc, A., \& Isern, J. 1996, ApJ, 465, L27.

Rauch, T. 1997, A\&A, 320, 237.

Starrfield, S., Truran, J., Sparks, W., \& Arnould, M. 1978, ApJ, 222, 600.

Williams, R.E. 1992, ApJ, 376, 721. 